trimester of pregnancy none of the other examined factors was associated with an increased risk. Birth asphyxia was not a significant risk factor.

\title{
EPILEPTIC ACQUIRED APHASIA
}

The syndrome of acquired aphasia, dementia and behavior disorder in a child with partial complex epilepsy and continuous spike and waves during sleep is reported from the Service de Pediatrie, et Service de Neurologie, CHUV, Lausanne, Switzerland. The child was observed from age three to 18 years and deterioration in behavior and language occurred between the ages of $3-1 / 2$ and 5 years, coinciding with the period of maximal EEG activity and the presence of almost continuous spike waves during sleep (CSWS). Improvement coincided with the disappearance of CSWS, between the ages of $5-1 / 2$ and 6-1/2 years, and the onset of a unilateral focus that persisted throughout the following years. A definite correlation between the paroxysmal EEG abnormalities and the neuropsychological deterioration and improvement was established retrospectively. (Roulet E, Deonna T et al. Acquired aphasia, dementia, and behavior disorder with epilepsy and continuous spike and waves during sleep in a child. Epilepsia July/August $1991 ; \underline{32}: 495-503)$.

COMMENT. Complex partial seizures with recurrence in adolescence and the persistence of an epileptic focus on the EEG are atypical features of the Landau-Kleffner syndrome but this diagnosis could not be definitely excluded.

\section{RETT SYNDROME}

\section{CEREBRAL LIPIDS IN RETT SYNDROME}

The lipid membrane composition of cerebral tissue from five patients, age 12-30 years, and from 14 age-matched controls was studied at the Department of Psychiatry and Neurochemistry and Pediatrics, University of Goteborg, Goteborg, Sweden. A selective loss of myelin-associated lipids and an enrichment of gangliosides was demonstrated in the temporal white matter. The ganglioside pattern showed an increase of astroglial cell-associated gangliosides and reduced proportions of gangliosides GD1a and GT1b. The fatty acid compositions of ethanolamine phosphoglyceride, choline phosphoglyceride, and galactosylceramide were normal. (Lekman AY, Hagberg BA, Svennerholm LT. Membrane cerebral lipids in Rett syndrome. Pediatr Neurol May/June 1991; $\underline{7}: 186-190)$.

COMMENT: There is as yet no specific biochemical marker for Rett syndrome. These changes in lipid membranes are thought to be secondary, confirming the neuropathologic finding of slight demyelination in Rett syndrome. PET studies performed on six patients with Rett syndrome at the Division of Child Neurology, National Center Hospital for Mental Disorders, Kodaira, Tokyo, Japan showed that 